\title{
Floor and Motion Classifying Scheme Exploiting Smart-phone for Indoor Movements
}

\author{
Younghoon Kim, Nammoon Kim and Youngok Kim \\ Department of Electronic Engineering, Kwangwoon University, 447-1Wol gye- \\ dong, No won-gu, Seoul, 139-701, Republic of Korea \\ kimyoungok@kw.ac.kr
}

\begin{abstract}
In these days, smart-phone becomes a major personal communication device and more and more hardware including various sensors as well as software including various applications tend to be mounted on it. In this paper, we propose a classification scheme that classifies ambulatory movements of the user and the floor where the user exists with a smart-phone. In the proposed scheme, ambulatory movements of the user are classified by exploiting the barometer and the accelerometer, while the GPS data is used to classifies the floor information. With the proposed scheme, various motions, such as walking, stop, up and down motions with elevator or stairs, are distinguished by utilizing the data from the barometer and the accelerometer. When the user enters a building, the building information is confirmed with the GPS information and the smart-phone can classify ambulatory movements and the floor information of the user. An Android application is developed for the performance evaluation of the proposed scheme. The proposed scheme is evaluated with experiments at general building in university and the accuracy of the proposed scheme is more than $94 \%$ for floor estimation, although there is still works to improve the estimation of movements.
\end{abstract}

Keywords: Barometer, Accelerometer, Ambulatory movement, GPS, Rotation matrix

\section{Introduction}

Lately, many kinds of smart-phones are released. More and more hardware including various sensors as well as software including various applications tend to be mounted on the newly released smart-phone [1]. Each smart-phone has various sensors for its own purpose, but the barometer, the accelerometer, and GPS receiver are commonly mounted. Especially, GPS is most popular navigation system in outdoor environments. However, it is hard for GPS to estimate exact locations and altitudes under non-line-of-sight (NLOS) conditions with satellites, likewise indoor environments [2]. Signal strength and accuracy of GPS also are significantly decreased in indoor and underground conditions. When GPS information is not reliable, other sensors such as the barometer and the accelerometer can replace the roll of GPS for indoor navigation [3-5]. In this paper, we propose a classification scheme that classifies ambulatory movements of the user and the floor where the user exists with a smart-phone. When the user with a smart-phone enters a building, the signal to noise ratio (SNR) of GPS is significantly decreased. When the SNR of GPS is decreased, the GPS information can be utilized to decide which building the user enters at that moment. With the information of building, the proposed scheme can classify ambulatory movements and floors of the user more accurate. In conventional classification scheme, ambulatory movements and floors can be classified with energy detection which needs calculations of high complexity [6]. In the proposed scheme, ambulatory movements of the user are classified by exploiting the barometer and the accelerometer. The sensor drift that causes errors, and compensate errors $[7,8]$ of barometer is also considered in the proposed scheme. With 
rotation matrix, it is possible to convert accelerometer datum into vertical components [9], [10]. By employing the rotation matrix scheme, the proposed scheme can detect ambulatory movements and floors regardless of smart-phone direction. An Android application is developed for the performance evaluation of the proposed scheme and the proposed scheme is evaluated with experiments at general building in university. The rest of paper is organized as follows. Section 2 represents the related works of proposed scheme and provides the detailed description of the proposed scheme. In Section 3, the performance of the proposed scheme is evaluated with experiments. Finally, concluding remarks are given in Section 4.

\section{System Description}

The flow chart of the proposed scheme is shown in Figure 1. Based on the received GPS information, firstly it detects the nearest building and gets the information of the building from the data base.

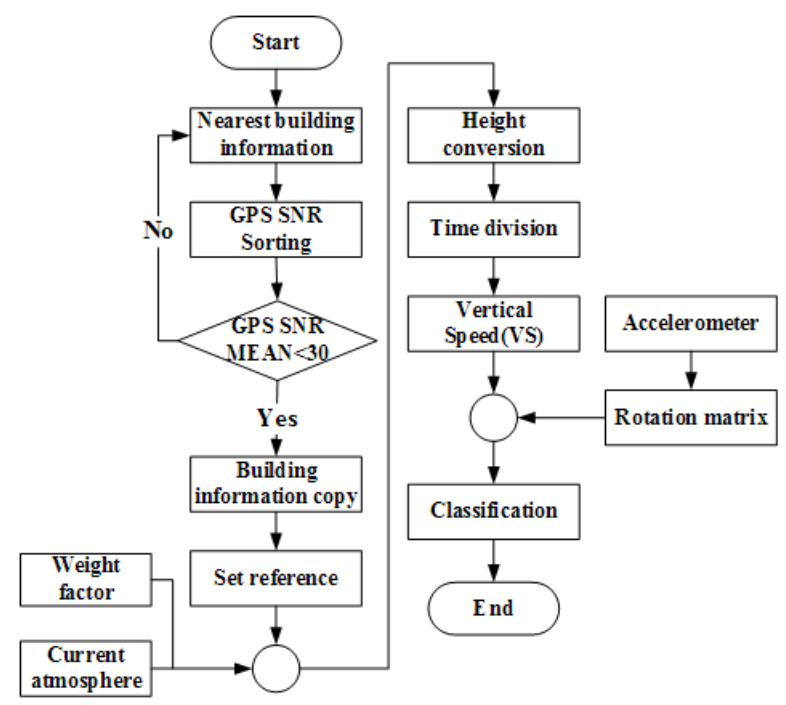

Figure 1. Flow Chart of the Proposed Scheme

When many satellites are detected, it is necessary to sort SNRs of GPS signals from satellites. In the proposed scheme, the 5 largest SNRs from the sorted GPS-SNR are utilized. The mean value of 5 largest SNRs is used to distinguish whether the user is located in outdoor and indoor conditions. If the mean value is over the threshold of GPSSNR, the user is considered to be located in outdoor conditions and the smart-phone detects the information of building. Since high value of GPS-SNR is more reliable than low value of GPS-SNR, the device updates the information of the nearest building in high SNR. In case of low GPS-SNR, it copies the latest information that contains the height of a floor for the nearest building. Meanwhile, it sets last atmosphere value that is detected before entering into the indoor condition as a reference atmosphere. When the current height is calculated by the current atmosphere and the reference atmosphere, weight factor is multiplied because of the sensor drift for the barometer. The smart-phone used in the experiments saves 40 samples and detects sensor values at the rate of $20 \mathrm{~Hz}$. Vertical speed (VS) is derived from the saved samples of the current height and the sensor rate. Acceleration variance (AV) can be derived from the rotated sensor data of the accelerometer. The AV detects the vibrations of vertical direction. By applying the threshold to the VS and the AV, ambulatory movements can be classified with the proposed algorithm. 


\subsection{Rotation Matrix}

A rotation matrix is utilized to obtain the vertical components among each axes of the accelerometer. In Euclidean space, the rotation matrix can be used as rotation. Actually, orientation operating in earth centered inertial coordinate system can be calculated by the gravity direction in the body frame. Therefore, the rotation matrix, $\mathrm{R}$, can be defined as follows:

$R=\times\left[\begin{array}{ccc}\cos \psi \cos \theta & \sin \psi \cos \theta & -\sin \theta \\ -\sin \psi \cos \theta+\cos \psi \sin \theta \sin \phi & \cos \psi \cos \phi+\sin \psi \sin \theta \sin \phi & \cos \theta \sin \phi \\ \sin \psi \sin \phi+\cos \psi \sin \theta \cos \phi & \sin \psi \sin \theta \cos \phi-\cos \psi \sin \phi & \cos \theta \cos \phi\end{array}\right]$

where $\phi, \theta, \psi$ represent three rotation angles, which are shown in Figure 2. The transformed accelerometer data can be obtained by multiplying the rotation matrix and the accelerometer data. The sum of the transformed three axes component means the vertical component. In the proposed scheme, it also is regarded as the vertical component.

\subsection{Height Conversion}

The height conversion can be derived from the equation that calculates absolute altitude by both atmospheres of sea level and current location, and it can be expressed as follows:

$$
\begin{gathered}
h_{c}=h_{b}-\frac{R \cdot T_{b} \cdot \ln \left(P_{c} / P_{b}\right)}{g_{0} \cdot M} \\
h=h_{c}-h_{r}=-\frac{R \cdot T_{r} \cdot \ln \left(P_{c} / P_{r}\right)}{g_{0} \cdot M}
\end{gathered}
$$

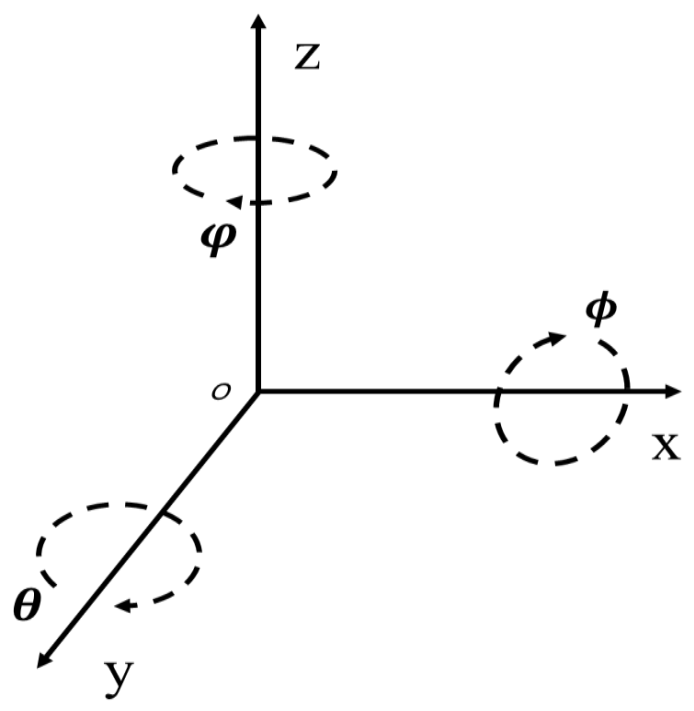

Figure 2. Rotation Angles

where $h_{c}$ is height at current location, $h_{b}$ is height at the sea level, $h_{r}$ is height at reference location, $h$ is the difference of height between the current and the reference location, $\mathrm{R}$ (= 
$8.31432 \mathrm{~N} \cdot \mathrm{m} /(\mathrm{mol} \cdot \mathrm{K}))$ is universal gas constant, $T_{b}(=288.15 \mathrm{~K})$ is the standard temperature at sea level, $T_{r}$ is the temperature at the reference location level, $P_{c}$ is the pressure at the current location level, $P_{b}$ is the pressure at the sea level, $P_{r}$ is the pressure at the reference location level, $g_{0}\left(=9.80665 \mathrm{~m} / \mathrm{s}^{2}\right)$ is the gravitational acceleration constant , and $M(=0.0289644 \mathrm{~kg} / \mathrm{mol})$ is the molar mass of air of earth.

The equation (2) is used to convert from the current atmosphere to the current altitude. By replacing the sea level to the reference value, the difference of height, (3), between the current and the reference location can be derived from the ratio of reference atmosphere to current atmosphere. The current floor can be estimated roughly by the equation (3) if the height of a floor is known.

\subsection{Drift Compensation}

By subtracting the estimated heights from the real heights, the error can be calculated. To minimize the error, the appropriate value for compensation is defined through the experiments. Note that the weight for compensation should be considered whether the reference atmosphere is larger than the current atmosphere or not in general.

Figures 3 and 4 show the comparison between the estimated height and the real height. As shown in the figures, the sensor drift are detected. If the estimated value is less than real value in positive side, weight value should be larger than 1 to increase value. However, weight value should be less than 1 to increase value, when the estimated value is less than real value in negative side.

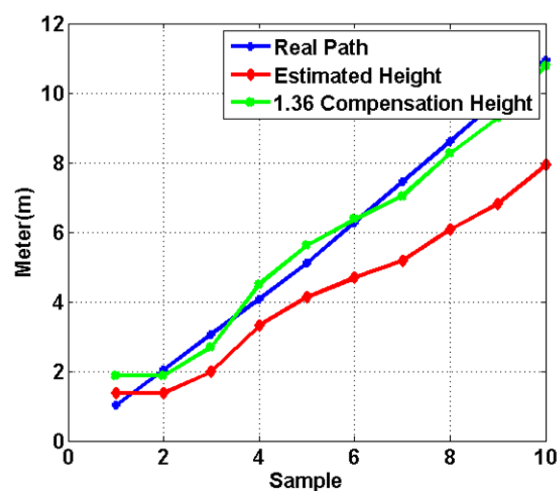

Figure 3. Comparison between the Estimated Height and the Real Height In Positive Side

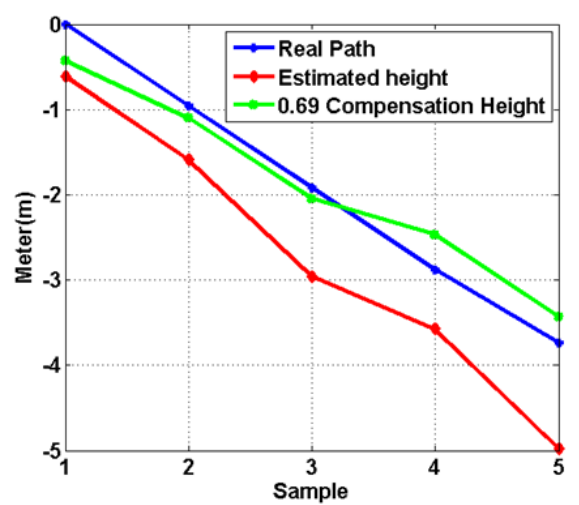

Figure 4. Comparison between the Estimated Height and the Real Height In Negative Side 


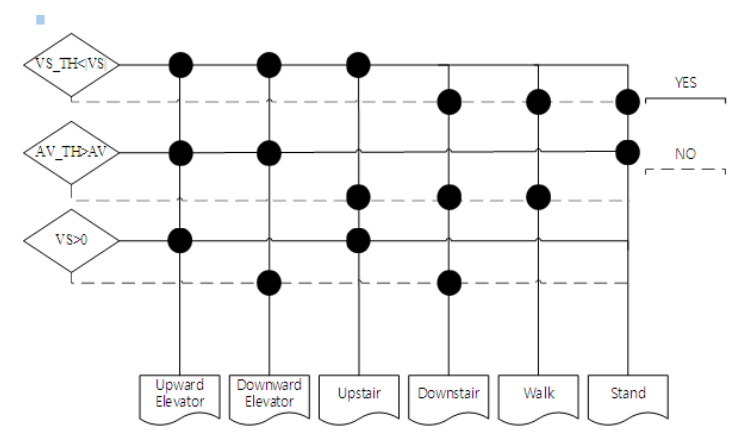

Figure 5. Classification Algorithm by Using the Thresholds, Where VS_TH Is the Threshold of Vs and AV_TH Is the Threshold of Av

When the reference atmosphere is larger than the current atmosphere, in the proposed scheme, the weight is defined as 1.36 , while the weight is defined as 0.69 in the other case. Note that the value for each position is the averaged value of 100 samples at $20 \mathrm{~Hz}$ sampling rates. The weighted heights are calculated as follows:

$$
\begin{aligned}
& h_{w}=1.36 \times h \\
& h_{w}=0.69 \times h
\end{aligned}
$$

where $h$ is the difference of height between the current and the reference location.

\subsection{Vertical Speed}

Vertical speed can be used to distinguish vertical movements from other movements. A ratio of $h_{w}$ to time is defined as the vertical speed and is expressed as follows:

$$
V=\frac{h_{w}}{t}
$$

where $v$ is velocity in period of $h_{w}, \mathrm{t}$ is time in period of $h_{w}$. Time of collecting data can be calculated by dividing total samples by sampling rates. The device saves the 40 samples of sensor data at sampling rate, which is $20 \mathrm{~Hz}$. Equation (6) indicates sign of speed that determined direction of upwards and downwards. The Equation (6) also can be used to distinguish vertical movements via stairs or elevator from horizontal movements, walking, and standing motion. If the equation (6) is positive, it means that the user moves upward direction. In contrast of upward direction, downward direction has negative sign of (6). Details of proposed classification scheme in Figure 1 are shown in Figure 5. With the velocity threshold and the AV threshold, as shown in the Figure 6 kinds of ambulatory movements can be distinguished. Movements of walk and stand are independent with the sign of estimated height and the VS, because those are horizon movements.

\subsection{Variance of Vertical Accelerometer Component}

As a result of rotation matrix, vertical component can be calculated. In the proposed scheme, 40 samples are saved at $20 \mathrm{~Hz}$ rates and the variance is obtained with the saved samples. The AV can be expressed as the result of calculating vertical component of accelerometer. Since the AV indicates the variance of vertical movements, the AV can detect how the user vibrates to the vertical direction. Therefore, it can figure out two different types of movements. The one is for walking, movements via stairs, the other is for standing, movements via elevator. 


\section{Experiment Results}

The proposed scheme is evaluated with experiments at general building in university and a general smart-phone of $G$ series of S company was used. Figure 6 shows the top view of Haw do building of our university and its entrance as a starting point of experiments. When the user enters the building, the value of GPS-SNR is decreased rapidly. Thus the starting point based on the GPS signal is regarded as the starting position of the experiments. Note that the starting point is obtained from GPS signal and it was averaged with 500 samples. For convenience and realistic results of experiments, an Android application is developed to detect the motions of the user and the floor where the user is located. Figure 7 shows a screen shot of the developed Android application program. As shown in the Figure, 6 different movements and the estimated floor information are displayed in the screen.

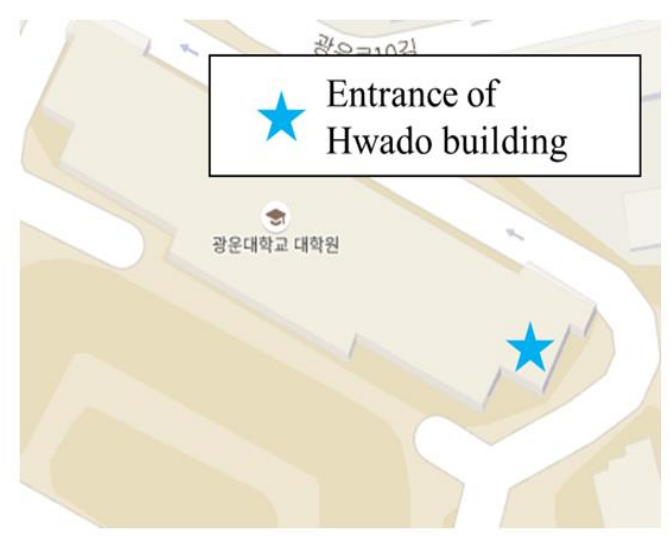

Figure 6. Top View of Hwado Building and its Entrance as a Starting Point of Experiments

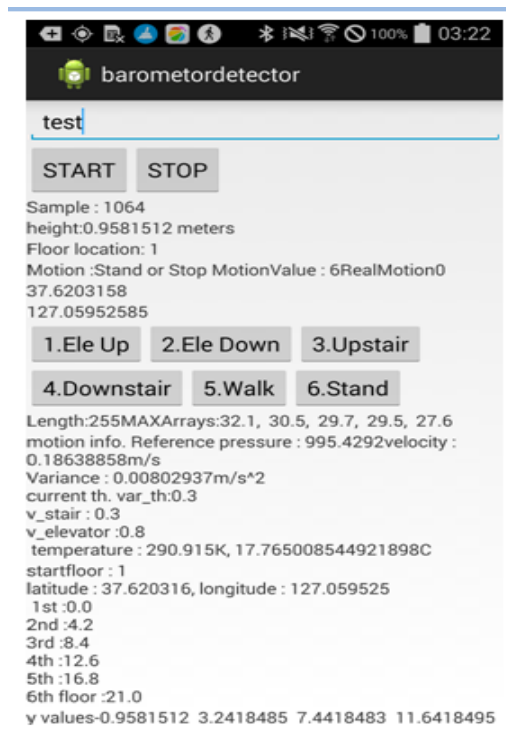

Figure 7. Screen Shot of the Developed Android Application Program

\subsection{GPS-SNR Decision}

In Table 1, the 5 largest values of GPS-SNR for various situations, such as outdoor, indoor, and under a tree, are detected and compared. Note that the value of SNR is a mean of 500 samples and is ordered in terms of larger value. As shown in the table, the values 
of GPS-SNR are rapidly decreased by moving from outdoor to inside of the building. Since the mean of the 5 largest values of GPS-SNR is used in the proposed scheme, 30 is determined as the threshold of GPS-SNR, although there is a degradation of SNR under a tree condition.

Table 1. 5 Largest Values of GPS-SNR for Various Situations

\begin{tabular}{|c|c|c|c|c|c|c|c|}
\hline \multicolumn{2}{|c|}{ Location } & SNR1 & SNR2 & SNR3 & SNR4 & SNR5 & Mean \\
\hline \multicolumn{2}{|c|}{ Outside } & 38.7594 & 36.7392 & 35.1160 & 33.8068 & 32.2600 & 35.3363 \\
\hline \multirow{2}{*}{ Hwado } & $10 \mathrm{~cm}$ in & 34.5322 & 27.9026 & 25.0758 & 21.4280 & 18.5328 & 25.4942 \\
\cline { 2 - 8 } & $50 \mathrm{~cm}$ in & 30.3934 & 23.2982 & 21.1508 & 21.2920 & 20.7548 & 23.3778 \\
\hline \multicolumn{2}{|c|}{ Under a tree } & 35.0198 & 34.0774 & 33.3154 & 31.1708 & 30.1696 & 32.7506 \\
\hline
\end{tabular}

\subsection{Threshold Decision}

Table 2 shows the variance of each motion in terms of the VS and the AV over 800 samples. As shown in the table, the VS of elevator is the largest and the VS of stairs is second one, while the $\mathrm{AV}$ of stairs is the largest and the $\mathrm{AV}$ of walking is second one. It is natural when the motions of real life are considered. Since the signs of upwards direction and downwards direction are different, the absolute value of speed components was used. Through the extensive experiments, the thresholds of VS and AV are defined as: 0.31 in AV, 0.32 in VS of stair, and 0.82 in VS of elevator. Note that they are the half of sum between standing and walking in AV, walking and stairs in VS, stairs and elevator in VS.

Table 2. Variance of each Motion in Terms of the VS and the AV

\begin{tabular}{|c|c|c|c|c|}
\hline & Standing & Walking & Stairs & Elevator \\
\hline VS & 0.1584 & 0.2218 & 0.4114 & 1.2282 \\
\hline AV & 0.0316 & 0.5891 & 0.9043 & 0.0278 \\
\hline
\end{tabular}

\subsection{Experiment Path}

Figure 8 shows the front view of Hawdo building and the path of experiment. As shown in the figure, the user starts at the start point of the $1^{\text {st }}$ floor, moves via $6^{\text {th }}$ floor, and arrives at the end point of the other side of $1^{\text {st }}$ floor. In detail, the user steps up via stairs to $3^{\text {rd }}$ floor, and then the user moves from $3^{\text {rd }}$ floor to $4^{\text {th }}$ floor by the elevator. After getting out at $4^{\text {th }}$ floor, the user gets in the elevator again and moves to $6^{\text {th }}$ floor. In $6^{\text {th }}$ floor, the user walks to the opposite side and then steps down via stairs to $5^{\text {th }}$ floor. From $5^{\text {th }}$ floor, the user moves to $3^{\text {rd }}$ floor by the elevator and steps down via stairs to $2^{\text {nd }}$ floor and then walks for a while in $2^{\text {nd }}$ floor. Finally, the user steps down via stairs to $1^{\text {st }}$ floor and the user stops at the end point.

\subsection{Results}

The experiments are performed with 6 different users and repeated 22 times. Experimental results are divided into each motion and are expressed as conditional probability. Abbreviation of each motion is defined as: UP is to move upwards via elevator, DO is to move downwards via elevator, US is upstairs, DS is downstairs, W is walking, $\mathrm{S}$ is standing. 


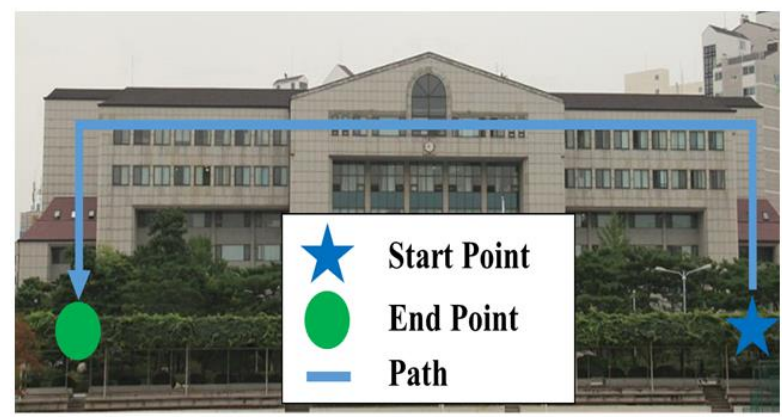

Figure 8. Front View of Hawdo Building and the Path of Experiment

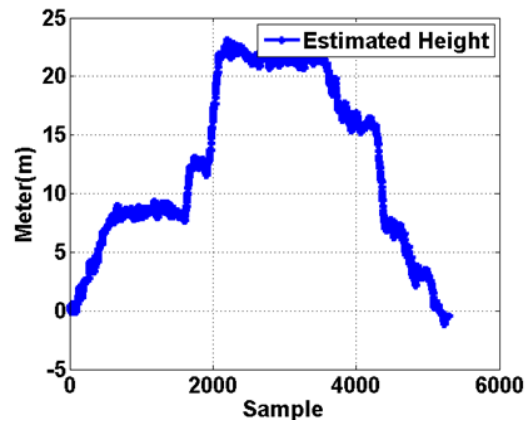

Figure 9. Estimated Height, HW

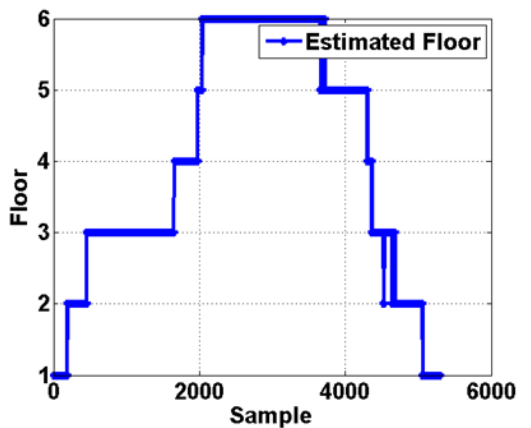

Figure 10. Estimated Floor based on Estimated Height, HW

\subsubsection{Floor Detection}

The floor where the user is located is determined by using the estimated heights, $h_{w}$, which can be obtained from the equations (4) and (5). Figure 9 shows the estimated height. As shown in the figure, fluctuation of barometer occurs and it can cause errors [11]. Figure 10 shows the estimated floor. As shown in the figure, the proposed scheme can classify the floor clearly without fluctuation.

\subsubsection{Motion Detection}

Motions are classified by the proposed algorithm, which is shown in Figure 5. In the experiments, as shown in Figure 7, the motions are classified as numbers. In the Figure 1 is to move upwards via elevator, 2 is to move downwards via elevator, 3 is upstairs, 4 is downstairs, 5 is walking, 6 is stand, respectively. The results of motion estimation are shown in Figure 11. As shown in the figure, the real motions are well matched by the proposed scheme. 


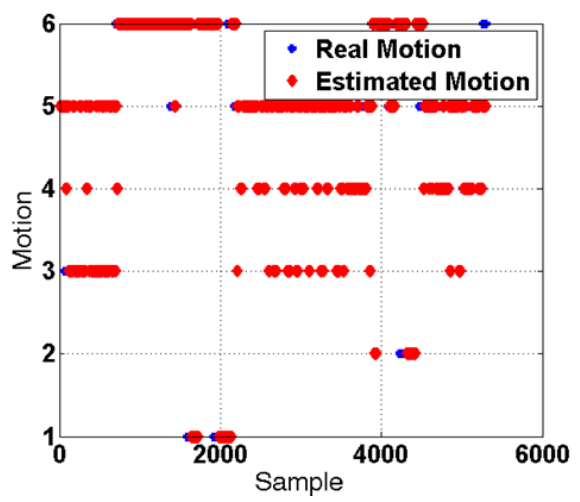

Figure 11. Comparison between Real Motions and Estimated Motions

\subsubsection{Performance Evaluation}

Performance evaluation is expressed as conditional probability in Tables 3 and 4. As shown in the table 3, the floor estimation is accurate as high as $94 \%$. However, the motion estimation is not good as the floor estimation because there is alternative motion, which causes the error. By investigating the results in table 4, for example, it is noticed that the conditional probability P (UP|UP) is slightly higher than P (S|UP), while the conditional probability of other motions is negligible. Note that similar situations occurs for $\mathrm{P}$ (DO|DO), P (US|US), P (DS|DS), P(W|W) cases.

In case of elevator and stairs, error rate is too high. First reason is the delay of determination. When device determines the motion, it uses 40 samples of saved sensor data. 40 samples mean 2 seconds. Second reason is the mistake of experimenter. For example, in case of elevator, each experimenter decided elevator motion at different time. Some recorded the elevator motion while they are waiting for upwardness or downwardness in elevator. Others recorded the elevator motion when elevator starts to move. In case of stairs, stairs are not straight. It means that they should walk for a while when experimenter moves upstairs or downstairs. Time of stairs and elevator motion is shorter than walking and stand. The time delay and the mistake of experimenter can be critical for the estimation of motions during short time. According to the Figure 11, the smart-phone matches most of real motions, while it shows the errors of delay.

Table 3. Conditional Probability of Floor Estimation

\begin{tabular}{|c|c|c|c|c|c|}
\hline $\mathrm{P}(1 \mid 1)$ & $\mathrm{P}(2 \mid 1)$ & $\mathrm{P}(3 \mid 1)$ & $\mathrm{P}(4 \mid 1)$ & $\mathrm{P}(5 \mid 1)$ & $\mathrm{P}(6 \mid 1)$ \\
\hline 1 & 0 & 0 & 0 & 0 & 0 \\
\hline $\mathrm{P}(1 \mid 2)$ & $\mathrm{P}(2 \mid 2)$ & $\mathrm{P}(3 \mid 2)$ & $\mathrm{P}(4 \mid 2)$ & $\mathrm{P}(5 \mid 2)$ & $\mathrm{P}(6 \mid 2)$ \\
\hline 0.0418 & 0.9582 & 0 & 0 & 0 & 0 \\
\hline $\mathrm{P}(1 \mid 3)$ & $\mathrm{P}(2 \mid 3)$ & $\mathrm{P}(3 \mid 3)$ & $\mathrm{P}(4 \mid 3)$ & $\mathrm{P}(5 \mid 3)$ & $\mathrm{P}(6 \mid 3)$ \\
\hline 0 & 0.05296 & 0.9470 & 0 & 0 & 0 \\
\hline $\mathrm{P}(1 \mid 4)$ & $\mathrm{P}(2 \mid 4)$ & $\mathrm{P}(3 \mid 4)$ & $\mathrm{P}(4 \mid 4)$ & $\mathrm{P}(5 \mid 4)$ & $\mathrm{P}(6 \mid 4)$ \\
\hline 0 & 0 & 0 & 1 & 0 & 0 \\
\hline $\mathrm{P}(1 \mid 5)$ & $\mathrm{P}(2 \mid 5)$ & $\mathrm{P}(3 \mid 5)$ & $\mathrm{P}(4 \mid 5)$ & $\mathrm{P}(5 \mid 5)$ & $\mathrm{P}(6 \mid 5)$ \\
\hline 0 & 0 & 0 & 0 & 1 & 0 \\
\hline $\mathrm{P}(1 \mid 6)$ & $\mathrm{P}(2 \mid 6)$ & $\mathrm{P}(3 \mid 6)$ & $\mathrm{P}(4 \mid 6)$ & $\mathrm{P}(5 \mid 6)$ & $\mathrm{P}(6 \mid 6)$ \\
\hline 0 & 0 & 0 & 0 & 0.02241 & 0.9775 \\
\hline
\end{tabular}


Table 4. Conditional Probabilities of Each Motion

\begin{tabular}{|c|c|c|c|c|c|}
\hline $\mathrm{P}(\mathrm{UP} \mid \mathrm{UP})$ & $\mathrm{P}(\mathrm{DO} \mid \mathrm{UP})$ & $\mathrm{P}(\mathrm{US} \mid \mathrm{UP})$ & $\mathrm{P}(\mathrm{DS} \mid \mathrm{UP})$ & $\mathrm{P}(\mathrm{W} \mid \mathrm{UP})$ & $\mathrm{P}(\mathrm{S} \mid \mathrm{UP})$ \\
\hline 0.5386308 & 0 & 0.00372655 & 0 & 0 & 0.45764262 \\
\hline $\mathrm{P}(\mathrm{UP} \mid \mathrm{DO})$ & $\mathrm{P}(\mathrm{DO} \mid \mathrm{DO})$ & $\mathrm{P}(\mathrm{US} \mid \mathrm{DO})$ & $\mathrm{P}(\mathrm{DS} \mid \mathrm{DO})$ & $\mathrm{P}(\mathrm{W} \mid \mathrm{DO})$ & $\mathrm{P}(\mathrm{S} \mid \mathrm{DO})$ \\
\hline 0 & 0.5449252 & 0 & 0 & 0 & 0.4550747 \\
\hline $\mathrm{P}(\mathrm{UP} \mid \mathrm{US})$ & $\mathrm{P}(\mathrm{DO} \mid \mathrm{US})$ & $\mathrm{P}(\mathrm{US} \mid \mathrm{US})$ & $\mathrm{P}(\mathrm{DS} \mid \mathrm{US})$ & $\mathrm{P}(\mathrm{W} \mid \mathrm{US})$ & $\mathrm{P}(\mathrm{S} \mid \mathrm{US})$ \\
\hline 0 & 0 & 0.5311224 & 0.03618153 & 0.4268493 & 0.00058466565 \\
\hline $\mathrm{P}(\mathrm{UP} \mid \mathrm{DS})$ & $\mathrm{P}(\mathrm{DO} \mid \mathrm{DS})$ & $\mathrm{P}(\mathrm{US} \mid \mathrm{DS})$ & $\mathrm{P}(\mathrm{DS} \mid \mathrm{DS})$ & $\mathrm{P}(\mathrm{W} \mid \mathrm{DS})$ & $\mathrm{P}(\mathrm{S} \mid \mathrm{DS})$ \\
\hline 0 & 0 & 0.02640108 & 0.5712162 & 0.4014447 & 0.0009379509 \\
\hline $\mathrm{P}(\mathrm{UP} \mid \mathrm{W})$ & $\mathrm{P}(\mathrm{DO} \mid \mathrm{W})$ & $\mathrm{P}(\mathrm{US} \mid \mathrm{W})$ & $\mathrm{P}(\mathrm{DS} \mid \mathrm{W})$ & $\mathrm{P}(\mathrm{W} \mid \mathrm{W})$ & $\mathrm{P}(\mathrm{S} \mid \mathrm{W})$ \\
\hline 0.00069903 & 0.00081479 & 0.1458343 & 0.1940476 & 0.5897526 & 0.068851579 \\
\hline $\mathrm{P}(\mathrm{UP} \mid \mathrm{S})$ & $\mathrm{P}(\mathrm{DO} \mid \mathrm{S})$ & $\mathrm{P}(\mathrm{US} \mid \mathrm{S})$ & $\mathrm{P}(\mathrm{DS} \mid \mathrm{U})$ & $\mathrm{P}(\mathrm{W} \mid \mathrm{S})$ & $\mathrm{P}(\mathrm{S} \mid \mathrm{S})$ \\
\hline 0.01912362 & 0.01593179 & 0.00925662 & 0.00454714 & 0.03630922 & 0.91483159 \\
\hline
\end{tabular}

\section{Conclusions}

In this paper, we proposed a classification scheme that classifies ambulatory movements of the user and the floor where the user exists with a smart-phone. In the proposed scheme, ambulatory movements of the user are classified by exploiting the barometer and the accelerometer, while the GPS data is used to classifies the floor information. With the proposed scheme, various motions, such as walking, stop, up and down motions with elevator or stairs, are distinguished by utilizing the data from the barometer and the accelerometer. An Android application is developed for the performance evaluation of the proposed scheme. The proposed scheme is evaluated with experiments at general building in university and the accuracy of the proposed scheme is more than $94 \%$ for floor estimation, although there is still works to improve the estimation of movements. According to the experimental results, it is shown that the motion estimation is not good as the floor estimation because there is alternative motion, which causes the error. However, this alternative motion can be distinguished by considering the position information of the user whether the user is near the elevator, stair, or in the aisle. Therefore, we plan to combine the proposed scheme with a positioning scheme as a future work.

\section{Acknowledgements}

This research was supported by the MSIP (Ministry of Science, ICT and Future Planning), Korea and NAVER Corp., under ICT/SW Creative research program supervised by the NIPA(National IT Industry Promotion Agency) (NIPA-2014-(H051114-1006)).

\section{References}

[1] J. Lee, Y. Chuah, and K. Chieng, "Smart Elderly Home Monitoring System with an Android Phone", International Journal of Smart Home, vol. 7, (2013), pp. 3.

[2] V. Zaliva and F. Franchetti, "Barometric and GPS altitude sensor fusion", 2014 IEEE International Conference on Acoustic, Speech and Signal Processing (ICASSP), (2014) May 4-9, Florence, Italy.

[3] A. Anjum and U. Ilyas, "Activity recognition using Smartphone sensors", 2013 IEEE Consumer Commun, and Networking Conference (CCNC), (2013) January 11-14, Nevada, USA.

[4] O. Yurur, C. H. Liu and W. Moreno, "Unsupervised posture detection by smart phone accelerometer", Electronics Letters, vol. 49, (2013), pp. 8.

[5] Y. Kim, S. Kim, D. Kang, H. Park, N. Kim, S. Yang and Y. Kim, "Study on Motion Recognition Scheme Using Acceleration Sensor in Mobile Devices", International Journal of Smart Home, vol. 7, (2013), pp. 6. 
[6] Y. Ohtaki, M. Susumago, A. Suzuki, K. Sagawa, R. Nagatomi and H. Inooka, "Movements and evaluation of energy consumptions utilizing accelerometers and a barometer", Microsystem Technologies, vol. 11, (2005), pp. 8.

[7] B. Li, "Using barometers to determine the height for indoor positioning", (2013) October 28-31, Montbeliard Belfort, France.

[8] R. L. Blanchard, "A New Algorithm for Computing Inertial Altitude and Vertical Velocity", IEEE Transactions on Aerospace, (1971).

[9] H. Rehbinder and X. Hu, "Drift-free attitude estimation for accelerated rigid bodies", Automatica, vol. 40, (2004), pp. 4.

[10] Y. Kim, N. Kim and Y. Kim, "A Classification Scheme of Ambulatory Movements with Barometer and Accelerometer of a Smart-Phone", The 3rd International Conference on Computer, Information and Application (CIA), (2015) May 21-23, Yeosu, Korea.

[11] K. Muralidharan and A. J. Khan, "Barometric phone sensors, more hype than hope", HotMobile 2014 Proceedings of the 15th Workshop on Mobile Computing Systems and Applications, (2014) February 26-27, USA.

\section{Authors}

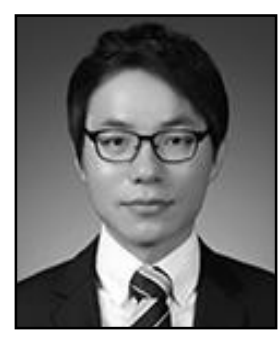

Younghoon Kim, received the B.S. degree in electronics engineering from Kwangwoon University, Seoul, Korea in 2014. $\mathrm{He}$ is currently working toward the M.S. degree at the Kwangwoon University. His research interests include motion recognition, indoor positioning techniques and systems.

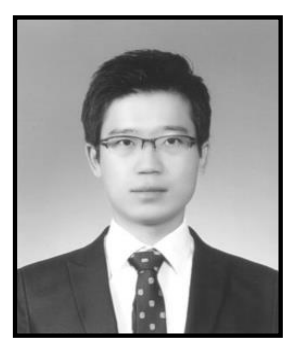

Nammoon Kim, received the B.S. degree in electronics engineering from Kwangwoon University, Seoul, Korea in 2011. He is currently working toward the Ph.D. degree at the Kwangwoon University. His research interests include ultra-wideband wire-less communication systems, high precision positioning techniques and systems.

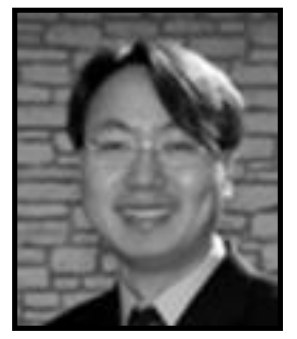

Youngok Kim, received the B.S. degree in mechanical engineering from Yonsei University, Seoul, Korea in 1999, and the M.S. and $\mathrm{Ph} . \mathrm{D}$. degrees in electrical and computer engineering from the University of Texas at Austin, Austin, in 2002 and 2006, respectively. From 2006 to 2008, he was a senior researcher at Infra Laboratory of Korea Telecom (KT), Seoul, Korea. In March 2008, he joined the Department of Electronic Engineering of Kwangwoon University, Seoul, Korea, as a faculty member. His research interests include ultra-wide band wireless communication systems, OFDM-based systems, precise ranging and location systems, PAPR reduction techniques, diversity techniques for wireless systems, and multipleaccess schemes in multicarrier systems. 
International Journal of Smart Home

Vol. 9, No. 7 (2015) 